\title{
Confinement Effects on the Collective Excitations in Thin Nematic Films
}

\author{
M. M. Wittebrood and Th. Rasing \\ Research Institute for Materials, University of Nijmegen, Toernooiveld 1, 6525 ED Nijmegen, The Netherlands
}

S. Stallinga

Philips Research Laboratories, Professor Holstlaan 4, 5656 AA Eindhoven, The Netherlands

\author{
I. Muševič \\ J. Stefan Institute, Jamova 39,61000 Ljubljana, Slovenia
}

(Received 27 March 1997)

\begin{abstract}
The influence of confinement on the spectrum of collective excitations in ultrathin nematic layers of octylcyanobiphenyl is studied, using quasielastic light scattering in planarly and hybridly aligned films. Both systems show confinement-induced relaxation rate gaps in the originally gapless spectrum of director fluctuations. On the mesoscopic scale, the magnitude of the relaxation rate gap scales as the inverse thickness of the nematic layer and can be very well described by a Landau-de Gennes theory, in which the boundary conditions play an important role. [S0031-9007(98)05324-1]
\end{abstract}

PACS numbers: 61.30.Eb, 68.10.Cr, 78.66.Qn

The question "What happens with the structure and dynamics of a liquid crystal near a confining surface?" is not only relevant for liquid crystal displays (LCD's). In a more general sense, the properties of a thin layer of liquid on a solid surface are relevant for the understanding of surface diffusion and melting, catalysis, lubrication, wetting, etc., in particular, when the thickness of the interfacial layer approaches molecular dimensions (see [1] and references therein). Because of the long-range orientational order in liquid crystals, the length scale where these confinement phenomena are expected to play a role is much larger than in normal liquids and therefore rather easily accessible.

The structural properties of liquid crystals (LC) in confined geometries have therefore recently attracted a lot of interest. For example, calorimetric and light scattering experiments in confined media indicate significant confinement-induced shifts and pretransitional phenomena at the $I \rightarrow N$ phase transition [2-6], and can be rather well described with a Landau-de Gennes phenomenological theory $[7,8]$. On the other hand, our knowledge of the dynamics of confined nematics [9-12] is rather scarce and inconsistent, since most of the studies were done on rather complex confined systems such as nematics in aerogels or porous matrices. In these systems the effects of confinement are intermixed with the effects of randomness and interpore interactions, which are inherent to these disordered systems. As a result, the observed dynamics is still a matter of controversy, since authors have reported a variety of dynamical phenomena, ranging from ultraslow [9] to ultrafast [10]. Very recently, important dynamical studies of more simple polar molecules in such porous media have been reported [1].

In this paper, we study finite size effects on the dynamics of collective excitations in liquid crystals confined in between two flat walls, separated by a mesoscopic distance. What is important and new in our experiment is that we are able to exclusively investigate the influence of a controllable confinement on the spectrum of the excitations, while the effects of disorder were eliminated. We have performed relaxation time measurements both on thin uniform planar and hybrid LC layers, using a quasielastic light scattering technique. We present clear experimental evidence that the confinement on a mesoscopic length scale induces forbidden relaxation rate gaps in the originally gapless spectrum of the director excitations. We can describe our experimental results perfectly by a Landau-de Gennes fluctuation theory, which takes the finite size, as well as the boundary conditions, into account. As an important additional result, we are able to directly determine the surface anchoring constants.

Let us briefly recall the predictions of the phenomenological Landau-de Gennes theory $[13,14]$ for a thin nematic film. In the one elastic constant approximation, and taking into account the Rapini-Papoular boundary conditions, the free energy density of a nematic film is [13]

$$
f=f_{\text {elast }}+f_{\text {surf }}=\frac{1}{2} K \sum_{\alpha=x, y, z}\left(\nabla n_{\alpha}\right)^{2}+f_{\text {surf }}
$$

with

$$
\begin{aligned}
f_{\text {surf }}= & -\frac{1}{2}\left[C_{1}\left(e_{1 \|} \cdot \mathbf{n}\right)^{2}+W_{1}\left(e_{1 \perp} \cdot \mathbf{n}\right)^{2}\right] \delta(z) \\
& -\frac{1}{2}\left[C_{2}\left(e_{2 \|} \cdot \mathbf{n}\right)^{2}+W_{2}\left(e_{2 \perp} \cdot \mathbf{n}\right)^{2}\right] \delta(z-d) .
\end{aligned}
$$

Here, $K$ is the elastic constant, $C_{1}$ and $C_{2}$ are the inplane (along the surface) and $W_{1}$ and $W_{2}$ the out-ofplane (perpendicular to the surface) anchoring constants of the director field at the confining walls at $z=0$ 
and $z=d$. The easy (i.e., preferred) directions of the director field at the walls are described by the two unit vectors $\mathbf{e}_{1}$ and $\mathbf{e}_{2}$, respectively. For the hybrid case there will be no in-plane anchoring at the free surface $\left(C_{2}=0\right)$ (see Fig. 1). The solutions of the hydrodynamic equations for small fluctuations of the director field around the equilibrium profile lead to two dispersion branches, the so-called bend-twist and the bend-splay modes. These are overdamped plane waves with a parabolic dispersion relation and are distinguished by their polarization properties [15]. In the following, we shall limit the discussion to the dynamics of the bend-splay mode, which is selected by our experimental geometry: $\mathbf{n}$ and $\mathbf{q}$ (almost) parallel to the sample and almost perpendicular to the incoming and scattered light beams [see Fig. 1(a)]. Because of the symmetry breaking by the confining surfaces, there are two orthogonal solutions for a given wave vector $\mathbf{q}$, one corresponding to out-of-plane and the other to in-plane fluctuations at the surface.

The confinement imposes restrictions on $q_{z}$, the wave vector component normal to the surface, and therefore only discrete values of $q_{z}$ are allowed in a confined system [13]. For very strong anchoring, or for thicknesses larger than the extrapolation length $L_{i}=K / C_{i}$, the allowed $z$ component of the wave vector equals $q_{z}=$ $(n+1) \pi / d$ [16], leading to a $1 / d^{2}$ dependence of the relaxation rates. On the other hand, for mesoscopic distances the dispersion relation for the lowest allowed value of $q_{z}$ is
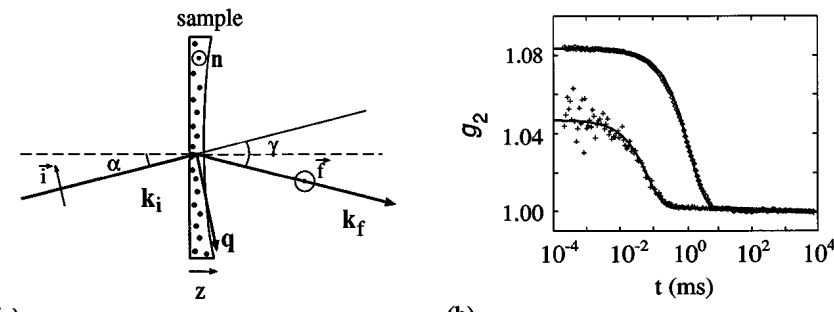

(a)

(b)

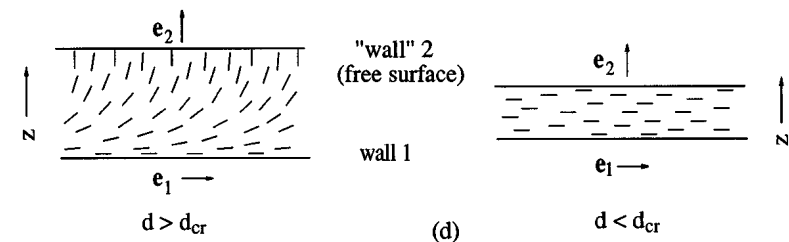

FIG. 1. (a) A schematic picture of the scattering geometry with the uniform planar cell as the sample. $\mathbf{k}_{i}$ and $\mathbf{k}_{f}$ denote the incident and scattering wave vector, respectively, with the polarizations $\vec{i}$ and $\vec{f}$. (b) Measured autocorrelation functions at $d=2.3 \mu \mathrm{m}(\diamond)$ and $d=0.12 \mu \mathrm{m}(+)$. Solid lines represent the best fit to a single, slightly stretched, exponential. The director orientations in thick (c) and thin (d) hybrid films (where $C_{2}=0$ and $W_{2} \ll W_{1}$ ). Wall 1 corresponds to the solid substrate and wall 2 corresponds to the air-crystal interface.

$$
\begin{aligned}
\tau^{-1}(\mathbf{q})= & \frac{K}{\gamma}\left(q_{x}^{2}+q_{y}^{2}\right)+\frac{1}{\gamma} \frac{C_{1}+C_{2}}{d} \\
& +\frac{1}{\gamma} \frac{C_{1} C_{2}-\left(C_{1}+C_{2}\right)^{2} / 3}{K}
\end{aligned}
$$

with $\gamma$ an effective viscosity.

In this regime, the confinement is therefore reflected in the onset of a forbidden relaxation rate gap that increases with the inverse thickness of the film. We have therefore a crossover from a $1 / d^{2}$ for a very strong anchoring to a $1 / d$ dependence at intermediate anchoring. The increase of the nonequilibrium free energy due to a director fluctuation is then comparable to the surface anchoring energy. Therefore, by measuring the thickness dependence of the relaxation rates at a fixed wave vector, one can also directly measure the average of the two surface anchoring constants $C_{i}$, which is of great technological importance.

We have measured the spectrum of the director excitations in ultrathin films of octylcyanobiphenyl (8CB) in homogeneous planar and hybrid aligned films using photon autocorrelation spectroscopy. With this method, one measures, at a given scattering wave vector $\mathbf{q}$, the autocorrelation function of the light intensity, scattered by thermal fluctuations of the director field. Because the dielectric tensor $\underline{\epsilon}(\mathbf{r}, t)$ depends linearly on the tensor $\mathbf{n}(\mathbf{r}, t) \otimes \mathbf{n}(\mathbf{r}, t)$, any fluctuation of the director also represents a fluctuation of the dielectric tensor, and this gives rise to a strong scattering of light [15]. In our experiment, we have used a scattering geometry that selects pure splay excitations ( $\mathbf{n} \perp \mathbf{q})$, and the scattering wave vector was chosen perpendicular to the confining direction $z$ (see Fig. 1). We calculated the scattering wave vector using the following values for the ordinary and extraordinary refractive indices: $n_{0}=1.5$ and $n_{e}=1.6$. Our geometry selects the fluctuations with relaxation rates given by Eq. (3). All experiments were done in the heterodyne regime, due to the mixing of the signal with the elastically scattered light from imperfections.

As a light source we used an argon-ion laser at a wavelength of $514 \mathrm{~nm}$. The light was focused onto the sample, irradiating an area of about $50 \mu \mathrm{m}$. The experiment was performed in the nematic phase at $T_{N I}-$ $T \approx 2 \mathrm{~K}$. The planar nematic films were prepared by gently pressing a long focal length glass lens $(f=2 \mathrm{~m})$ against a flat quartz plate. This created a "wedge-type" cell (12 mm in diameter) with a continuously variable thickness between 0.04 and $8 \mu \mathrm{m}( \pm 0.04 \mu \mathrm{m})$. Both surfaces were coated with a thin layer of polyvinyl alcohol (PVA) that was unidirectionally rubbed to give a welldefined planar surface anchoring with parallel orientations of the molecules at both confining surfaces. The cell was filled using capillary action deep in the isotropic phase. The hybrid layers were prepared by depositing $8 \mathrm{CB}$ on a nylon coated glass substrate using isopropanol as a solvent. Flat, pancakelike droplets (several mm 
in diameter) were formed in this way. Whereas the nylon surface aligns the $8 \mathrm{CB}$ molecules parallel to the surface, the molecules tend to align perpendicularly to the free surface. As a result, a hybrid aligned nematic film is formed, which is highly strained. The thickness of these hybrid films was varied by varying the $8 \mathrm{CB}$ concentration in the 8CB-isopropanol solution. For the thicker films, the thickness was determined by counting the interference, Newton rings. Because this is not possible for very small thicknesses, the thickness in this regime was calculated using the deposited amount of bulk material and measuring the size of the film area. The accuracy of this approach was checked by using it also for the thicker films.

The measured autocorrelation functions can be fitted by a single (slightly stretched) exponential function $\exp \left[-(t / \tau)^{s}\right]$ for all thicknesses, with $s$ ranging from 1.0 to 0.90 . Typical autocorrelation traces are given in Fig. 1(b) for two thicknesses. Note that even for the smallest thickness $(d=0.12 \mu \mathrm{m})$ there is no significant tail for longer delay times, indicating the absence of randomness in our well-defined geometry. (For $d \leq 0.1 \mu \mathrm{m}$, the autocorrelation signal became too small and noisy.)

The dispersion relation in the uniform cell is plotted in Fig. 2 for different thicknesses. The dispersions

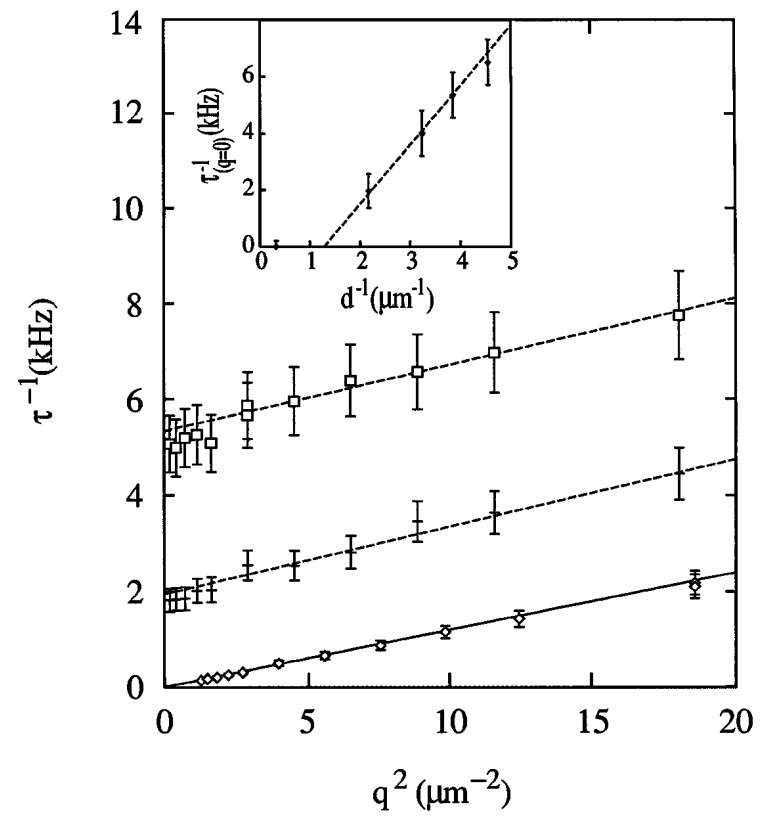

FIG. 2. The measured dispersion relations $\tau^{-1}\left(q^{2}\right)$ for different 8CB thicknesses in a uniform planar cell $[d=3 \mu \mathrm{m}(\diamond)$, $d=0.44 \mu \mathrm{m}(+)$, and $d=0.26 \mu \mathrm{m}(\square)]$. The solid line gives the dispersion relation of the bulk relaxation time with $K / \gamma=(1.3 \pm 10 \%) \times 10^{-10} \mathrm{~m}^{2} \mathrm{~s}^{-1}$. The dashed lines show the dispersion relations for thin films according to Eq. (3). The inset shows the $1 / d$ dependence of the relaxation rates extrapolated to $q=0$. The dashed line represents the best fit to Eq. (3) with $C_{\mathrm{PVA}}=(3.4 \pm 20 \%) \times 10^{-5} \mathrm{~J} / \mathrm{m}^{2}$. are clearly parabolic, as expected for a dissipative, overdamped system, and the calculated value of the coefficient $K / \gamma=(1.3 \pm 10 \%) \times 10^{-10} \mathrm{~m}^{2} \mathrm{~s}^{-1}$ leads to a $\gamma$ of $(3.4 \pm 10 \%) \times 10^{-2} \mathrm{Pas}$ (where we used $K=4.4 \mathrm{pN}$ [17]). This value of $\gamma$ is close to the bulk values, reported by others $[18,19]$. For a $3 \mu \mathrm{m}$ film, the relaxation rate of the excitations goes to zero for $q \rightarrow 0$ leading to a gapless bulk dispersion relation. For smaller thicknesses, however, a gap occurs at $q=0$ which increases for decreasing thicknesses. The inset in Fig. 2 shows the thickness dependence of this gap, which was obtained by extrapolating the dispersions to $q=0$. The data can be very well fitted to a $1 / d$ dependence for thicknesses smaller than $\approx 0.5 \mu \mathrm{m}$, giving the in-plane anchoring constant $C_{\mathrm{PVA}}\left(=C_{1}=C_{2}\right)=(3.4 \pm 20 \%) \times 10^{-5} \mathrm{~J} / \mathrm{m}^{2}$ for the $8 \mathrm{CB}$ molecules on rubbed PVA. This value is very close to the anchoring strengths reported by others and allows us to calculate the extrapolation length, which equals $0.14 \mu \mathrm{m}$ for this system. This means that the theoretical equation seems to hold up to thicknesses that are 4 times this extrapolation length. This seems surprising as Eq. (3) is strictly valid only for thicknesses much smaller than the extrapolation length. However, it appears that the approximated solution of the equations is quite close to the exact numerical solution for thicknesses up to 2 to 4 times the extrapolation length.

The inverse relaxation time as a function of inverse thickness is shown in Fig. 3 for both the uniform planar and the hybrid films. In both cases, we observe a constant bulk relaxation rate down to thicknesses of $\approx 1.7$ and $0.5 \mu \mathrm{m}$ for the planar and hybrid cell, respectively, and a clear increase in $\tau^{-1}$ for smaller thicknesses, which is in excellent agreement with the prediction of Eq. (3). Fitting the data to Eq. (3) and using the value for $K / \gamma$ from above, we find $C_{\mathrm{PVA}}=(3.5 \pm 20 \%) \times 10^{-5} \mathrm{~J} / \mathrm{m}^{2}$. This value agrees very well with the value obtained from the dispersion relation (Fig. 2).

In the hybrid case, we have a nonuniform director field for thicknesses larger than the critical thickness $d_{c r}=\left|K / W_{1}-K / W_{2}\right|$, with $W_{i}$ the out-of-plane anchoring constant of wall $i$ [14]. For $d<d_{c r}$, however, the director field becomes uniform with the director orientation determined by the stronger anchoring wall [see Fig. 1(d)]. Taking into account that the free surface aligns the molecules perpendicularly to the surface and has no in-plane anchoring, we can again apply Eq. (3) in this regime [13], but now in a simplified form with $C_{2}=0$. Figure 3 also shows the inverse relaxation time as a function of the inverse thickness for this case. Below a thickness $d_{c r} \approx 0.5 \mu \mathrm{m}$, a linear dependence is observed, as predicted by our theory. From these data and the value for $K / \gamma$, we can calculate the value for the in-plane anchoring constant of $8 \mathrm{CB}$ on nylon. We find $C_{\text {nylon }}=C_{1}=(3.4 \pm 30 \%) \times 10^{-5} \mathrm{~J} / \mathrm{m}^{2}$, which is equal to the value found for PVA. The experimentally determined value $d_{c r}$ is close to the value found in phase 


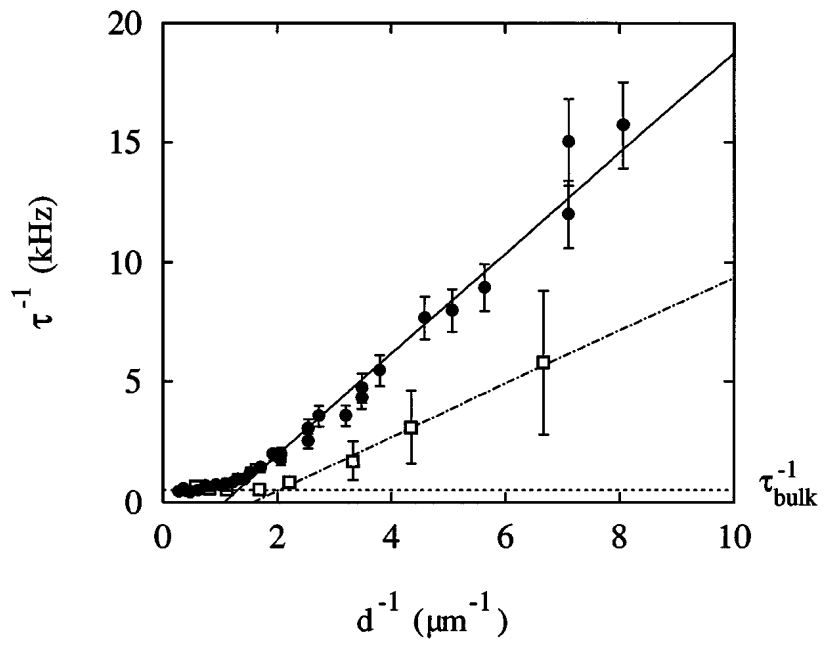

FIG. 3. The relaxation rate vs inverse layer thickness in the uniform planar $(\bullet)$ and the hybrid system ( $\square)$. The scattering wave vector $q$ was $(2.0 \pm 10 \%) \times 10^{6} \mathrm{~m}^{-1}$. The dashed line gives the bulk relaxation rate. The solid and dashed-dotted lines are fits to Eq. (3), with $C_{1}=C_{2}=(3.5 \pm$ $20 \%) \times 10^{-5} \mathrm{~J} / \mathrm{m}^{2}$ for the planar and $C_{1}=(3.4 \pm 30 \%) \times$ $10^{-5} \mathrm{~J} / \mathrm{m}^{2}, C_{2}=0$ for the hybrid system, respectively.

transition temperature measurements [5]. From $d_{c r}$, we can estimate the out-of-plane anchoring constant at the air-8CB interface $W_{2}=W_{\text {air }}$ (as $W_{2} \ll W_{1}$ [5]) and find $W_{\text {air }}=(0.9 \pm 30 \%) \times 10^{-5} \mathrm{~J} / \mathrm{m}^{2}$.

Our results show that, for both planar and hybrid nematic layers, the director dynamics is significantly influenced by the confinement. The good quantitative agreement with the Landau-de Gennes model also allows us to determine the in-plane anchoring strengths for $8 \mathrm{CB}$ at nylon and PVA surfaces, yielding $C_{\text {nylon }}=(3.4 \pm 30 \%) \times 10^{-5} \mathrm{~J} / \mathrm{m}^{2} \quad$ and $C_{\mathrm{PVA}}=(3.5 \pm 20 \%) \times 10^{-5} \mathrm{~J} / \mathrm{m}^{2}$. The similarity of these two anchoring constants is not surprising as both coatings are used in practice to give a very good planar alignment and have similar alignment characteristics. Because of the small number of experiments done in this field, comparison with anchoring constants found in the literature is difficult. We can, however, qualitatively compare our results to the dynamical measurements done in pores by extrapolation to smaller dimensions [13]. For thicknesses of 60 and $17.5 \mathrm{~nm}$, our extrapolated values are of the order of 60 and $15 \mu \mathrm{s}$, respectively. This is indeed close to the values of about 100 and $20 \mu \mathrm{s}$ (at $T_{N I}-T \approx 6 \mathrm{~K}$ ) found by Bellini et al. in $8 \mathrm{CB}$ in pores with sizes of 60 and $17.5 \mathrm{~nm}$, respectively [11].

In conclusion, we have determined, for the first time, the pure influence of confinement on the orientational dynamics in thin nematic films. We observe an onset of a forbidden relaxation rate gap in the originally gapless spectrum of the nematic director excitations. We find that the details of the thickness dependence of this relaxation rate gap depend on the boundary conditions and anchoring strengths of a liquid crystal at an interface and are described perfectly by our Landau-de Gennes fluctuation theory. This allows for a very accurate and straightforward determination of the in-plane surface anchoring constants, which are difficult to determine by other methods.

We would like to thank Dr. J. Dijkhuis for lending us the Malvern autocorrelator and A.F. van Etteger for technical support. Part of this research was supported by the PECO/Copernicus programmes ERBCIPACT930159.

[1] J.-P. Korb, L. Malier, F. Cros, Shu Xu, and J. Jonas, Phys. Rev. Lett. 77, 2312 (1996).

[2] L. Wu, B. Zhou, C. W. Garland, T. Bellini, and D. W. Schaefer, Phys. Rev. E 51, 2157 (1995).

[3] G. S. Iannacchione and D. Finotello, Phys. Rev. Lett. 69, 2094 (1992).

[4] N. A. Clark, T. Bellini, R. M. Malzbender, B. N. Thomas, A. G. Rappaport, C.D. Muzny, D.W. Schaefer, and L. Hrubesh, Phys. Rev. Lett. 71, 3505 (1993).

[5] M. M. Wittebrood, D. H. Luijendijk, S. Stallinga, Th. Rasing, and I. Muševič, Phys. Rev. E 54, 5232 (1996).

[6] For a recent review, see, for example, Liquid Crystals in Complex Geometries Formed by Polymer and Porous Networks, edited by G. P. Crawford and S. Žumer (Taylor \& Francis, London, 1996).

[7] P. Sheng, Phys. Rev. Lett. 37, 1059 (1976).

[8] T. J. Sluckin and A. Poniewierski, Mol. Cryst. Liq. Cryst. 179, 349 (1990).

[9] X-L. Wu, W. I. Goldburg, M. X. Liu, and J.Z. Xue, Phys. Rev. Lett. 69, 470 (1992).

[10] G. Schwalb and F. W. Deeg, Phys. Rev. Lett. 74, 1383 (1995).

[11] T. Bellini, N. A. Clark, and D. W. Schaefer, Phys. Rev. Lett. 74, 2740 (1995).

[12] F. M. Aliev and M. N. Breganov, Sov. Phys. JETP 68, 70 (1989).

[13] S. Stallinga, M. M. Wittebrood, D. H. Luijendijk, and Th. Rasing, Phys. Rev. E 53, 6085 (1996).

[14] G. Barbero and R. Barberi, J. Phys. (Paris) 44, 609 (1983).

[15] P. G. de Gennes, The Physics of Liquid Crystals (Clarendon, Oxford, 1974).

[16] K. Eidner, M. Lewis, H. K. M. Vithana, and D. L. Johnson, Phys. Rev. A 40, 6388 (1989).

[17] M. J. Bradshaw, E. P. Raynes, J.D. Bunning, and T.E. Faber, J. Phys. (Paris) 46, 1513 (1985).

[18] P. Martinoty, F. Kiry, S. Nagai, S. Candau, and F. Debeauvais, J. Phys. (Paris) 38, 159 (1977).

[19] F. W. Deeg, S. R. Greenfield, J. J. Stankus, V. J. Newell, and M. D. Fayer, J. Chem. Phys. 93, 3503 (1990). 